\title{
Yiming Shao, China CDC's Chief Expert of AIDS
}

\author{
Peter $\mathrm{Hao}^{1,8} ;$ Nankun Liu ${ }^{1,8} ;$ Zhenjun Li ${ }^{1}$; Jingjing $\mathrm{Xi}^{1, \ldots} ;$ Feng Tan ${ }^{1,4}$
}

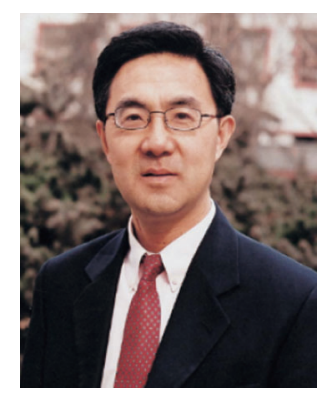

Yiming Shao is China CDC's Chief Expert of AIDS, the Chairman of the Academic Committee of the National Center for AIDS/STD Control and Prevention (NCAIDS), and the Vice President of the Chinese Microbiology Society (CMS) after chairing the CMS's Virology Committee (2001-2010). Shao graduated from Qingdao Medical College in 1983 and pursued a $\mathrm{PhD}$ at the Institute of Virology in the Chinese Academy of Preventive Medicine. He participated in the diagnosis of China's first cases of HIV and AIDS, isolated China's first HIV-1 virus, and developed the initial HIV diagnostic reagents for the country. After his PhD study, he served as the deputy director of the Department of HIV and director of the HIV Reference Laboratory in the Institute of Virology.

In 1989, Shao joined the World Health Organization (WHO) as a consultant to the Global Program on AIDS (GPA). Being assigned to develop a virus research program for GPA, Shao proposed to establish a WHO network for HIV isolation and characterization. He structured the network based on a " 3 by 3 " principle by studying HIV variation at 3 levels (genetic, immunological, and biological) and building 3 repositories to store biological samples and research reagents at the UK's National Institute of Biological Standards and Control (NIBSC) and the US's National Institutes of Health (NIH) and HIV sequences at Los Alamos Laboratory in the US. Shao's proposal was approved by GPA/WHO. He participated in the establishment of the WHO network by selecting the laboratories and drafting the initial guidelines with the help of Prof. Jay Levy of UCSF. The WHO HIV network became a major accomplishment for the WHO as it generated fundamental scientific data with numerous HIV sequences and research reagents to provide free access to the world's AIDS researchers for the development of AIDS diagnostic reagents, antiviral drugs, and vaccine research and development.

Shao's WHO experiences have also benefited his domestic work in China. He was credited for establishing the National AIDS Reference Laboratory and leading the National AIDS Laboratory Expert Committee to help China's Health Ministry to develop 3 continuously-used key infrastructures in the country: 1) the 5-level HIV testing network with over 40,000 laboratories for HIV testing and diagnosis since the mid-1990s; 2) the HIV molecular epidemiology network to trace the origin and spreads of the HIV strains since the late 1990s; and 3) the National HIV Drug Resistance (HIVDR) surveillance network to support the National Antiviral Treatment Program since the early 2000s. These research activities have trained thousands of laboratory staff of CDC networks and hospitals at the provincial, municipal, and county levels. These task forces and infrastructure provided strong technical support to China's AIDS diagnostic capacity, antiviral treatment, and HIV prevention.

In 1998, NCAIDS was built in CAPM by the Chinese government based on his HIV Reference Laboratory in the Institute of Virology and the AIDS Surveillance Center in the Institute of Epidemiology of CAPM. Shao served as the Deputy Director for Research and International Collaboration of NCAIDS and was the Founding Director for the National AIDS Reference Laboratory. Shao served as a lead expert in formulating China's first Mid- and Long-Term Plan for AIDS Prevention and Control and developed the National AIDS Control Goal by 2010. As the Deputy Representative in the Chinese Delegation to the United Nations General Assembly Special Session on HIV/AIDS (UNGASS), he provided technical facilitation to the Chinese delegation for the signing of the Declaration of Commitment on HIV/AIDS.

Shao's research areas include molecular epidemiology, drug resistance, immunology, and vaccinology. His team studied HIV genetic evolution, pathogenesis, and immune responses of the infected people, identified over $20 \mathrm{HIV}$ 1 clades and clusters, and discovered 7 new circulating recombinant forms (CRFs) of HIV-1. They mapped the transmission routes of all major HIV-1 strains in China and found that they were all CRF strains (CRF01, 07, 08, and 55), indicating the presence of high-risk behaviors. They recently discovered that different clusters of the same HIV-1 clade evolved different pathogenic paths with more virulent forms causing rapid immune deficiency and 
accelerated disease progression, which signaled the need to expand HIV surveillance from genotype-focused to phenotype-focused to provide earlier warnings for public health interventions.

Shao's team also worked to identify broader neutralizing antibodies and their generating mechanism in Chinese patients. Shao's team was the first to show that infusing anti-HIV cytotoxic T lymphocytes collected and amplified in vaccine immunized monkeys could suppress the rebound in simian-human immunodeficiency virus during ART interruption, which could indicate the possibility of developing an immune therapy strategy for a functional cure for HIV. His laboratory has develop into WHO's regional HIVDR laboratory to provided technical support to other developing countries in Asia and African.

Shao's team developed a novel HIV vaccine based on a) redesigned HIV immunogen, inspired by the first lentivirus (EIAV) vaccine developed by Chinese scientist and b) replication-competent vaccinia vector, derived from the Chinese smallpox vaccine. The DNA and vaccinia vaccines, complemented by priming and boosting regiments, achieved high protection rates against homologous challenges $(>85 \%)$ and heterologous challenges $(50 \%)$ in rhesus macaques. They have concluded 3 Phase I (Ia, Ib, Ic) and 1 Phase II clinical trials and induce both anti-HIV-1 antibody and T-cell responses. They are also preparing a Phase III trail Under a joint Sino-US HIV vaccine project, Shao's team's vaccines (DNA/vaccinia) and US NIH's vaccine (gp145) will be combined for clinical trials in China. Shao's group has published around 900 research papers in English and Chinese journals, and they also possessed 2 National Science and Technology Progressive Awards and numerous science awards by Health Ministry and Municipal governments.

Shao is an Adjunct Professor at Medical Schools of Beijing University, Zhejiang University and Nankai University. He served on several WHO Scientific Advisory Committees, including the Scientific and Technical Advisory Committee, HIV Vaccine Advisory Committee, HIVDR and on the Scientific Advisory Board of the Grand Challenge to Global Health from the Bill and Melinda Gates Foundation. He is currently serving in the Chinese Advisory Committee on Infectious Disease Control and Prevention, WHO's Product Development Vaccine Advisory Committee, and HIV Cure Advisory Committee of the International AIDS Society. He is a fellow of the American Academy of Microbiology.

As China CDC's Chief Expert of AIDS, Shao continues to conduct numerous investigations and to draft and submit many proposals to advise and guide the Chinese government. Shao's group served as a bridge between Chinese and international AIDS researchers through numerous domestic and international projects (EU, NIH, IDRC and BMGS etc.) he lead. He aims to strengthen AIDS and infectious disease control to promote public health education and related campaigns and to enhance international research collaborations and health aid to developing countries.

doi: $10.46234 / \mathrm{ccdcw} 2020.213$

\#Corresponding authors: Jingjing Xi, xijj@chinacdc.cn; Feng Tan, tanfeng@chinacdc.cn.

\footnotetext{
${ }^{1}$ Chinese Center for Disease Control and Prevention, Beijing, China.

\& Joint first authors.

Submitted: September 20, 2020; Accepted: September 25, 2020
} 